Ramírez M. Rafael F. Un caso más de embarazo ovárico llegado a término. Revista Médica, órgano de la Academia Nacional de Medicina.

Serpa Roberto. Embarazo extrauterino. Revista de la Facultad Nacional de Medicina. 1940.

Autores extranjeros:

Calatroni Carlos J. Terapéutica Ginecológica $4^{a}$ edición, 1946 .

Calderón Horacio y Molina V. Embarazo abdominal primitivo. Día Médico. 1946.

Charpentier A. Traité de laccouchement.

Conill Víctor. Embarazo ectópico. 2" edición, 1945.

rossen and Crossen. Diseases of women. ga edición.

urtis Arthur Hale. Ginecología. 1941
Davis Carl Henry. Ginecology and C. steirics. 1946.

Fabre. Manual de Obstetricia. 1941.

Coldberger Morris A. Ginecology surgery. 1942.

Hardy John A. Sinopsis of the diagnosis of the surgical diseases of the abdomen. $2^{2}$ edic., 1945.

Maygriery Schwabb. Manual de Obstetricia. 1943.

Novak. Ginecología. 1945.

Faradelo Carlos A. La histerosalpingografía en el diagnóstico del embarapo ectópico abdominal.

Pérez Manuel Luis. Tratado de obstetricia. 1943.

Recassens Girol. Tratado de Obstetricia.

Studdiford William E. Primary Peritoneal Pregnancy. An J. Obst. and Gin. Vol. 44,1942

\title{
Consideraciones sobre rupturas uterinas durante el año de 1949
}

\author{
Por EUDORO CASTIILO VEGA \\ Jefe de Clínica de Matemidad.
}

Ina de las complicaciones mús scrias que se pueden presentar en el e ercicio de la tocología es la ruptura i. erina.

Este accidente puede acontecer en el curso del embarazo o en el momento del trabajo; puede ser accidental $y$ raras veces espontáneo, pero casi simpre obedece a causa traumática. In la primera mitad del embarazo puede ser ocasionado por una gestación en la porción intersticial de la trompa, en un útero bicomio, en un útero in- fantil y en otras veces por excesiva invasión de la pared uterina por los elementos embrionarios, Al final de la gestación, el accidente por io general, está relacionado en los casos espontáneos, por la presencia de tejidos cicatrizales en la pared uterina, odelgazados progresivamente por la distensión creciente del órgano. Poroschin las ha atribuído a la ausencia relativa del tejido elástico; Jellinghaus y otros autores creen en la debilidad intrínseca de la pared uterina, por la 
formación excesiva de tejido conjuntivo en un punto vulnerado anteriormente. Casos hay debidos a una adenomiometritis difusa. Todas ellas son rupturas espontáneas y el lugar de su localización se halla con frecuencia en la porción superior del útero.

Por lo que respecta a las rupturas en el momento del pario que, son las que principalmente entraré a considerar, el contraste es muy claro, puesto que la ruptura está generalmente limitada al segmento inferior y los factores etiológicos son absoluiamente distintos. ción Matemo Infantil, sección del Frotesor Rodríguez Aponte, y sobre un total de 2.657 enfermas atendidas en el año de 1949, ningún niño se obtuvo vivo, en tanto que cinco madres sobrevivieron.

Estadísticas a m eric a n as arrojan sobre diez mil parios consecutivos, ctendidos en el New York Lying-In Hospital, solamente cuatro casos de ruptura uterina o sea una frecuencia de 1 por 2.500 partos. Presento el siguiente cuadro estadístico a modo de comparación en algunas materniáades mundiales:

\section{CIIN ICAS}

Cazos atendidos

Rupturas

Porcentajes

Keal Maternidad de Caridad de Londres
48.996

147.625 Similar a

$\begin{array}{rl}8 & 1 \times 6.124 \\ 84 & 1 \times 1.757\end{array}$

Wew York Lying-In Hospin
Eoston Lying-In Hospital

Flospital de Maternidad de Dublin.

Clínica de Maternidad de Moscú

Instituto de Protección Materno Infantil de Bogotá ..

Maternidad de Bucarest
57.412

118.581

2.657

23.016 al de New York.

\section{7}

77

$\begin{array}{rrr}34 & 1 \times 1.689 \\ 124 & 1 \times & 956\end{array}$

$\begin{array}{lll}1 \times & 379 \\ 1 \times & 300\end{array}$
Por lo que hace en relación al niño. raras veces permanece dentro de la cavidad de la matriz, sino que pasa $a$ la cavidad peritoneal y este tránsito es sinónimo de muerte fetal, una vez rotas las conexiones útero-placentarias.

La complicación en sí, es grave, y justamente el doctor Alfonso Fonnegra, en su tesis de grado así la calificó; con relativa frecuericia termina con muerte fetal y en muchas veces con la muerte materna. En los siete casos que observé en el Instituto de Protec-
Entre nosotros es frecuente y eso se cebe, sin duda alguna, al medio pobre, económicamente hablondo, y que por el mismo motivo, se acude a la atención obstétrica del parto a muje. res ignorantes y $\sin$ responsabilidad, que apellidándose enfermeras se comprometen a dirigir el trabajo del pario con las consecuencias que contemplamos, puesto que, es necesario aclarar que todos los casos de ruptura uterina de mis observaciones llegaron en este estado al Servicio de Matemidad. También conviene decir que si tales 
hechos acontecen en Bogotá, con cuanta mayor razón sucederán en nustros campos y provincias.

Debemos al tocólogo vienés Luirs Eandl (1842-1892), la explicación clara en lo tocante al modo de producirse; la etiología está totalmente relacionada con la doctrina del anillo de contracción y la formación anatomo-histolócica del segmento uterino interior. Durante el parto, al ser atraídos hacia arriba los haces musculares del cuello uterino por las contracciones enérgicas, el proceso de distensión del segmento se acentúa, hacia la parte activa del útero, acumulándose las fibras rousculares, mientras que en la parte pasiva se produce el adelgazamiento, Le se acentuará, si se presenta un ectado tetánico debido a un obstáculo en el paso: y en la misma forma indicada, el adelgazamiento puede avanzar y llegar a tal extremo que ceda facilmente el tejido y se desgarre la mairiz. Así lo explicaba Ruiz Contre:as, de Barcelona, en su tesis de grado "Estudio etiológico de la ruptura del ero grávido y consecuencias que de pueden deducirse como bases de ofilaxia y tratamiento".

In los casos que estudiamos hoy, las ipturas fueron por partos laboriosos era del servicio con niños grandes, or presentaciones transversas $\mathrm{m} x \mathrm{l}$ onducidas y abandonadas, y por emfleo poco juicioso de ocitócicos.

Es de notar igualmente que ninguno de los casos fue de ruptura espontánea sino todos consecutivos a mala concucta obstétrica; además, todas las upturas fueron completas, es decir, comunicadas con la cavidad abdomirali en cuanto a la dirección y forma de ellas, la mayoría fueron transversa- les en el segmento, alguna con extensión longitudinal hacia el cuello, coms se verá en el relato clínico-quirúrgico.

\section{Diagnóstico.}

El diagnóstico tue siempre fácil, derido al estado de abdomen agudo y sincopal, como llegaron las enfermas al Instituto, y a la fácil percepción del leto, como por debajo de la pared abcominal -a flor de piel- sin contar con el síntoma hemorragia y onemia aguda que nunca faltó. Sin embargo, en el caso de la historia particular de la Clínica Magdalena, el diagnóstico no fue hecho sino en el momento de intentar practicar un raspado como luégo se relate.

\section{Trciamiento.}

Existen aquí más que en cualquie: ctra entidad el tratamiento profiláctico $Y$ el tratamiento curativo.

Profiláctico. Un cuidado inteligente de la mujer en la gestación y en el trabajo: dirigiéndola desde el Consultorio Prenatal, hacia un hospital o clirica, para que cill sea atendida po: especiclista. Es conveniente saber apreciar suficientemente la conducta en una presentación de homioro y si todavía a la luz de la obstetricia mo. derna, es aconsejable la version por manicbras internas en vez de una cesárea. Contemplar con sabia sospecha la ílta de encajamiento de una presentación de vértice después de una hora de poderosas contracciones, al final del período de dilatación y mós aún, cuando el anillo de contracción comienza a ascender para saber cómo terminar el parto por medio obstétrico. quirúrgico. 


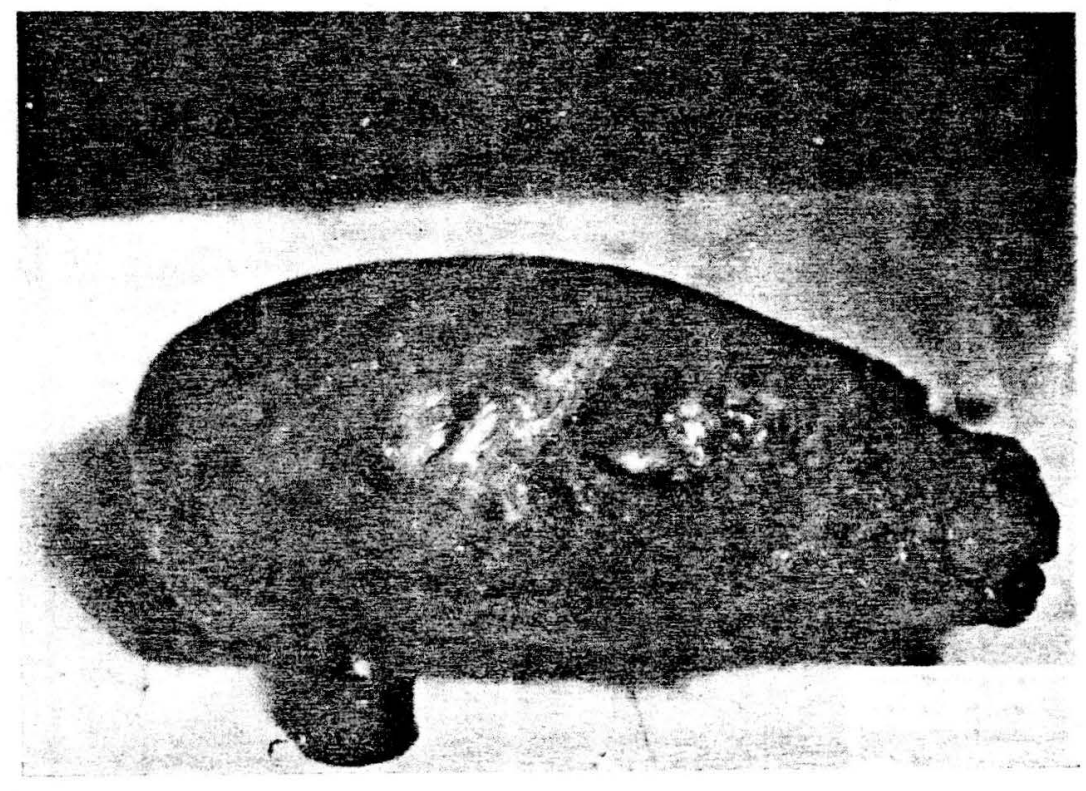

PIEZA DE AUTOPSIA

Watur rota y sutrada con cirugía conservadora, pero dicha sutura no prendió. 
Pertenece al tratamiento profiláctico, el aumento de los consultorios prenatales, para que las gestantes estén suficientemente informadas sobre los peligros de una mala atención obstétrica y se percaten una vez más, que no por el hecho de ser multigestantes no tienen nada que temer, como cs creencia general, pues la realidad estadística nos confiesa que las rupturas uterinas se presentan con mayor Irecuencia en las multigestantes y no en las primigrávidas. En los casos que presento a la consideración, no hubo ninguna primigestante, pero sí del segundo parto en adelante, hasta el ducdécimo. Es preciso acabar con la errórea creencia del vulgo que asevera que el primer parto es el difícil, y que la multiparidad garantiza la integricad biológica de la madre y del niño.

Anotamos igualmente que ninguna enferma de las que se complicaron con ruptura uterina, dió el antecedente de haber sufrido intervención cesárea onterior, ni intervención de legrado.

En cuanto a la edad, todas pasaron de los 35 años y solamente una fue de 23 años.

Curativo. En este campo existe gran divergencic de opinión sobre el trctamiento quirúrgico de la rupture uterina. La conducta seguida en los 3 casos presentados nos la dió el campo quirúrgico, sobre el que hubimos de actuar. El ideal sería el tratamiento quirúrgico conservador, pero desgraciadamente encontramos en la práctica la imposibilidad de la conservación integral del órgano y nos hemos visto precisados a practicar la Histerectomía subtotal con conservación de los anexos. Los resultados fueron satisfac- torios con el tratamiento drástico que fue menester ejecutar. Hubo una sutura del cuello uterino por ruptura del mismo con prolongación hacia el segmento, que terminó con defunción, dekida principalmente al estado de anemia y schock, al ingreso al hospital. De tres intervenciones conservadoras que fue posible realizar, terminaron dos con nuerte

\section{Causas en los casos presentados.}

Las causas de ruptura uterina en nuestros casos fueron así: por presentación de hombro abandonada fuera del servicio con niños grandes, tres casos. Por parto laborioso fuera del servicio y oplicaciones de ocitócicos en presentacjón cefálica, cuatro casos. Por maniobras de dilatación forzada fuera del servicio, un caso. Por maniobras abortivas en embarazo de tres meses, un caso. Todos, por mala atención obsiétrica: siete en el momento del parto y uno al querer interrumpir un embarazo. En un caso salió el producto de la concepción espontáneamente por via baja, en los demós se necesitó intervención para su extracción.

\section{Conducta para con el feto.}

\section{Los clásicos no definen claramente} ia conducta con el feto y las observaciones al respecto, entre nosotros, son muy variadas. Hay quienes opinan la extracción del feto, sea vivo o muerto por vía abdominal. Otros preconizem la extracción del feto por vía baja, por medio de la embriotomía La escuela ecléctica fue la seguida en el servicio, es decir, el caso impuso la conducta sin que ésta se pudiera predecir. Si la fresentación está encajada y por de- 
bajo del estrecho superior, cabe la embriotomía; con mayor razón cuando lo presentación está enclavada. Si la presentación está por encima del estrecho superior, ya sea intra o extravierina, debe abandonarse la extracción por vía vaginal porque fácilmente puede agrandarse la brecha uteri. na y dificultar más la intervención conservadora.

\section{Historias clínicas.}

Los siete casos presentados en el nomento del parto fueron observacioes en el servicio del Profesor Rodrívez Aponte. El caso de ruptura utena en la gestación fue obtenida an a Clínica La Magdalena, en mi cliensla particular.

Caso número 1. Historia número $\mathbf{4 4 0}$. ombre, H. B. Edad 37 años. VI gestaon. Fecha de ingreso, marzo 7 de 49. Fecha de salida, marzo 18 de 4. Causa de salida: curación.

Antecedentes generales, sin imporncia.

Antecedentes obstétricos: cinco niños término, vivos, normales. Causa de reso: parto laborioso fuera del serio desde hace dos días con presención deflejada y no encajada. Feto male muerto. (Es enviada al servipor un médico).

Examen actual: estado general, grave. Vientre en desarrallo que corresponde a un embarazo a término. Orgenos genitales varicosos y edematosos. Enferma obesa.

Altura uterina: 32 centímetros. $M a-$ triz de consistencia dura. Situación iongitudinal. Contracciones fuertes.

Datos fetales: número 1. Presentacien cefálica. Derecha. Por encima del estrecho superior. Ruídos negativos.
Tacto vaginal: vulva edematizada $y$ varicosa. Vagina amplia. Cuello borrado y dilatado co npletamente. Membranas rotas. Hay una brecha enorme en la región posterior del segmenti.

Tratamiento. Se aplica basiotribo para extraer el niño muerto y se practica el alumbramiento artificial. Luégo de aplicar tonicardiacos, transfusión ¿e 500 centímetros, suero Beclisin 500 c. c., se interviene por laparotomia media. Al abrir la cavidad abdominal se localiza una gran brecha en la cara posterior del segmento y abundantes coágulos organizados entre el miometrio y el peritoneo. Se hace histerectomía subtotal conservando los anexos Se aplicar 10 gramos de sulfa $y$ se cierra.

Anestesia: gases. Duración: hora y inedia.

Niño: único, femenino de 3.200 gramos, intrauterino, muerto.

Caso número 2. Historia número 517 Nombre T. Ch. Edad 38 años. VII gestación. Fecha de ingreso, marzo 17 de 1949. Fecha de salida, abril 3 de 1949 Causa de salida, curación.

Antecedentes genercles, $\sin$ importancia.

Antecedentes obstétricos: seis partos anteriores a término, normales, niños vivos. Causa de ingreso: parto con trabajo laborioso de tres días fuera del servicio, niño grande.

Examen actual: enferma angustiosa. Anémica. Pulso 100 por minuto.

Tensión arterial, 10 por 6. Sudores fríos. Hipotermic.

Examen obstétrico: abdomen duro, corresponde a una altura de 29 centirnetros. Forma en corazón de noipe. Auscultación negativa. 
Tacto vaginal. Vulva edernatosa. Gran desgarro en la pared anterior con dirección hacia la derecha dei segmento y vagina. Cuello con borrariento incompleto. Sangre viscosa $\mathrm{y}$ negruzca. Membranas rotas. Presentación de pelvis completa.

Tratamiento Después de tonificar suficientemente a la enterma con suero dextrosado y plasma, se logra ex. traer por vía vaginal fácilmente un felo de tamaño grande y muerto. Peso 4.040 gramos, masculino y en principio de maceración.

Comprobada la brecha después del ciumbramiento artificial se procede a practicar una laparotomía media. Se trata de suturaria brecha de segmento, pero se encuentra luégo con la gran brecha de la vagina y por lo tanto se decide la histerectomía para hacerlas más accesibles. Se sutura con caigut número 3 y se peritoniza. Se deja sulfa en solución y se cierra dejando ovarios libres.

Anestesia: gases. Duración: una hora. Se pasa transfusión durante la iniervención.

Niño: masculino. Unico, de 4.040 gramos. Muerto intraútero.

Caso número 3. Historia número 690. Nombre, R. M. Edad 39 años. XII gestación. Fecha de ingreso, abril 7 de 1949. Fecho de salida, abril 7 de 1949. Causa de salida, defunción.

Examen actual. La enferma llega al servicio a las 9 a. m. del día 7 de abril, cespués de prolongado trabajo de Farto fuera del hospital. Manifiestan ios familiares que había sido somet:di a repetidas maniobras de "dilatación" del cuello y múltiples aplicaciores de ocitócicos. Es recibida por la enfermera de turno, quien presencia la expulsión de un teto de 3.020 gramos de peso, con una circular y muerto. El alumbramiento tiene lugar inmediatamente seguido de una hemo. rragia grave con estado sincopal y síntomas de anemia aguda. Acude el Jete de Clínica, quien comprueja una enorme brecha que comienza al lacio izquierdo del cuello uterino y se extiende hacia el segmento inferior. I.uéso de aplicar piasma, 250 centímetros Y tónicos generales, se procede a lcr sutura por vía vaginal de la brecha con pequeña anestesia de éter-oxígeno. Se sutura en surcete con catgiti número 2. Se tapona la matriz y el segmento con mecha grande impregrada de sulfa. Desgraciadamente la enterma fallece acto seguido, por onemia aguda.

Anestesia: Eter-oxígeno. Duración: un cuarto de hora.

Niño: único, masculino de 3.020 gramos. Muerto.

Caso número 4. Historia número 1.660. Nombre, A. V. Edad 23 años. IV gestación. Fecha de ingreso, agosto 13 de 1949. Fecha de salida, agosto 13 de 1949. Causa de salida, defunción.

Examen cbstétrico. Al llegar al servicio se sabe que la enferma es traída de Turmequé. Tensión arterial: no se encuentra. Pulso 130. Temperatura: 35 grados. Membranas rotas. Hemorragia sí hay. Embarazo a término. Diámetros: bilíaco: 21 centímetros. Bicrestal: 24 centímetros. Bitrocanterino: 29 centímetros. Baudelocque: 17 centimetros. Matriz contracturada.

Datos fetales: único. Situación transversa. Presentación de hombro A. I. D. Dorso anterior. Anota la paciente que desde el día 10 de agosto a las 4 a. m. comenzó el trabajo de parto 
y que el día 12 a las 15 horas apareció un brazo fuera de la vagina y que en estas circunstancias una "comadrora", se puso a traccionar por el brazo procidente sin lograr la salida del feto.

Tratamiento. Después de las aplicaciones de tónicos generales de rutina, se pasa a la cirugia, donde se le prac. tica una embriotomía.

Después del alumbramiento se procede a revisar la matriz encontrándose una gran brecha en el segmento inferior y en una longitud de 15 centimetros. Después de nueva transfusión sanguínea 200 c. c., se hace una laparctomía, comprobándose lo anteior, encontrándose gran cantidad de oágulos ya organizados y iétidos. anca reacción peritoneal con necrcs de los tejidos vecinos al útero. Se cide histerectomía, pero la enferma hece.

Anestesia: Eter-oxígeno.

Miño: único. Masculino de 3.100 gramos de peso. Muerto.

\section{Caso número 5. Historia número} 712. Nombre, M. C. G. Edad 48 años: gestación. Fecha de ingreso, agosto de 1949. Fecha de salida, septiem2 de 1949. Cousa de salida, cuion.

La enterma llega al servicio en esdo sincopal y abdomen agudo. Heorragia negra vaginal y percepción el feto como por debajo de la pared dominal. Tensión arterial de 9 x 4 . 4lso 112. Situación longitudinal. Prentación cefálica. Posición izquierda. uscultación negativa. La enferma osivo atendida por "comadrona" y por in "médico", quienes ejercieron fuercomprensión sobre el abdomen desvués de aplicarle varias inyecciones de ocitócicos. Llega a las 24 horas, pero desde las 16 no siente el feto. Desde las 14 horas tiene vómito, a las 18 horas lipotimia y desde las 19, no tiene más dolores. Se hace el liagnóstico de ruptura uterina.

Tratamiento. Teniendo en cuerta las condiciones anotadas como llego la paciente y pensando que la práctica de la embriotomía previa a la intervención por vía abdominal no era aconsejable, tanto por el tiempo que Ella ocupaba, como por la posibilidad de ampliar la brecha uterina ya exis. tente, se decide la intervención por vía abdominal. Al hacer la laparotomía se aprecia la presencia de sangre $\epsilon$ n la cavidad abdominal; se comprue. ba el diagnóstico clínico por cuanto que a nivel del segmento se ve una brecha horizontal de unos 15 centímetros de longitud. Por esa brécha, se "extrae el feto muerto y se practica el alumbramiento artificial. Creyendo que no existía otra brecho se procede a la sutura cuidadosa de la matriz, pero luégo se comprueba una nueva bre. cha er sentido longitudinal en la cora posterior y que llega hasta la vecindad de la vagina. Como esta sutura resulta mixy laboriosa debido a la irreGularidad de los bordes y a la poca firmeza de los mismos, se procede a practicar una histerectomía subtotal, con conservación de los anexos. Se ceja penicilina y sulfa en la cavidad Y se cierra la pared en los tiempos crdinarios.

Anestesia: Ciclo-éter oxígeno.

Niño: único. Masculino de 3.600 graros. Muerto.

Caso número 6. Historia número 1.968. Nombre, F. R. Edad 35 años. VIII gestación. Fecha de ingreso, septiem- 
bre 22 de 1949. Fecha de salida, 5 de cotubre de 1949. Causa de salida, culración.

Manifiesta la enferma que los dolores comenzaron a las 6 a. m., del 21 de septiembre, atendida por "enferriera", fuera del servicio. A las 2.2 horas de ese día y estando con contracciones fuertes y subintrantes sintió bruscamente un dolor intenso en el hipogastrio, seguido por la abolición de las contracciones, presencia de hemorragia vaginal y ausencia de movimientos fetales. En estas condicioneis es trasladada al Hospital.

Examen clínico. Se aprecian los síntomas antes enunciados y a la palpación se encuentran partes fetales $\alpha$ flor de piel. Auscultación negativa. Coágulos sanguíneos en la cavidad vaginal. Cuello con dilatación de 8 centímetros, blando, delgado y las membranas rotas.

Tratamiento. Al hacer la laparotomía se comprueba la ruptura uterina. Se halla el feto fuera de la matriz, dentro de la cavidad peritoneal. Hay un gran desgarro transversal sobre la cara anterior del segmento uterino $y$ sobre sus bordes. Se resuelve practicar la histerectomia subtotal. Se deian 5 gramos de sulfa en polvo dentro del peritoneo: Sangre y plasma duronte la intervención.

\section{Anestesia: Gases.}

Niño: único. Femenino de 3.600 gramos de peso. Muerto

Caso número 7. Historia número 2.373. Nombre D. R. Edad 38 años. V sestación. Fecha de ingreso, noviembre 18 de 1949. Fecha de salida, 30 de 1949. Causa de salida: defunción. por peritonitis generalizada, necrosis del ciego, bronconeumonía y nefritis aguda, según rt'ato de autopsia.

Llega al servic o con una presentación de hombro abandonada. La enferma manifiesta que tuvo dolor interso desde la víspera y hemorragia vacinal. A la palpación se aprecian parins fetales en la covidad abdominal.

Tratamiento. Laparotomía por vía media. Se extrae un feto muerto de la cuvidad peritoneal. Se verifica el alumbramiento. Se resuelve practicar ia sutura conservadora de la matriz en las brechas desgarradas. Se deja suiia líquida en la cavidad peritoneal y se cierra.

Anestesia: Eter-oxígeno. Plasma y transfusión durante la intervención.

Niño: único. Masculino de 3.600 gramos. Muerto.

\section{Caso número 8. Historia}

(CIinica "La Magdalena").

El resumen es el siguiente: fue llevada la enferma a la Clínica, con el cbieto de que se le practicara un raspado uterino por aborto incompleto. La enierma presentaba un cuadro clinico de anemia intensa, pero sin síntoma alguno de abdomen agudo. Discreta hemorragia vaginal.

Tratamiento. Como el cuello preser:taba cierto grado de dilatación, se posó la bujía número 10 y se notó con sorpresa que penetró con suma facilidad y a una profundidad no de acuerdo con el tamaño de la matriz. Se pasa una cureta roma y antes de practicar raspado alguno, se advierte que penetra profundamente. Se trata de hacer un raspado uterino en la matriz sin encontrar resto alguno para extraer. Se hace, pues, el diagnóstico tardio de una ruptura uterina de! 
cuerpo por maniobra abortiva criminal. Se inierviene por vía alta. comprobándose la perforación uterina del fondo. Previa limpieza y avivamiento de los bordes de la brecha se hace la sutura con catgut número l cromado y con puntos separados. Luégo otra rueva en surcete y con el mismo catgut número 1. Se procura peritoniza: esta sutura a expensas del peritoneo visceral. Se aplica sulfa en polvo y se cierra.

Anestesia: Ciclo-oxígeno.

Post-operatorio: sin accidentes. Sale por curación.

\section{Resumen y conclusiones.}

De los 8 casos presentados, pertenecen los 7 primeros a rupturas uterinas n embarazo a término y con motivo le! trabajo, y el otro a maniobra aborva criminal en el tercer mes de gesrción. Desde luége todas ellas occionados por mala conducta obstéica.

Las brechas en su mayoría fueron a el segmento uterino inferior, transarsales unas, longitudinales otras $Y$ ztas en algún caso. Del mismo odo las rupturas se presentaron con urtos laboriosos y fetos grandes, siexo el menor de todos de 3.100 gramos peso.

Todos los niños estabon muertos $a$ llegada al servicio. Fueron unos exaídos por vía vaginal, otros por vía bdomina!.
Tres casos fueron por presentaciores de hombro mal conducidas o abandonadas; dos casos por administraciones inadecuadas de ocitócicos: un caso por maniobra de dilatación rnal ejecutada y un caso por parto laborioso de una duración mayor a 40 licras. En cucnto al tratamiento seguido, se practicaron cinco histerectomias subtotales con conservación de los anexos; cuatro de las pacientes sclieron por curación y la otra por defunción (caso número 4), debida a! estadio extremadamente grave ce la enferma a su llegada al servicio. $A$ los tres carsos restantes se les practicó sutura conservadora con el resultccio de dos defunciones y una curación.

Dol balance final sacamos la conclusión que la mejor conducta cuirúrgica no es ni la histerectomía ni 15 sutura conservadora, sino aquelia que. en el momento quirúrgico esté indicaja a) criterio del cirujano; $\sin$ embargo, por la experiencia habida en las ne. cropsias realizadas, nos dimos cuento h del difícil afrontamiento de brechos irregulares y el poco éxito de dichas suturas por perfecta y cuidadosa qua la operación haya sido ejecutado. No debemos olvidor el inmenso beneficis que nos prestó und oportund transin. sion o un benéfico plasma en el pre. operatorio, durante la intervención y en el post-operatorio.

Mayo 4 de 1950. 